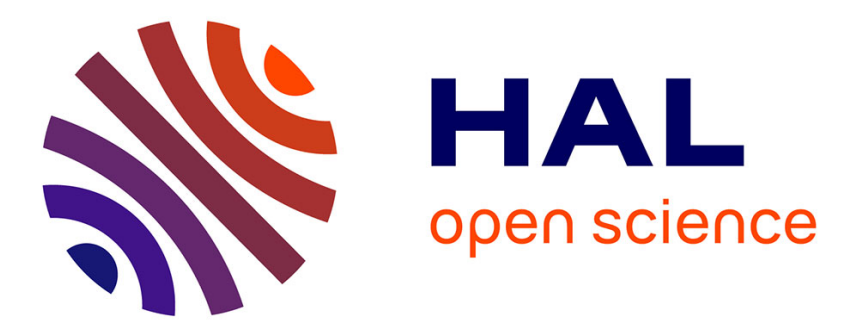

\title{
Control of Lipase Enantioselectivity by Engineering the Substrate Binding Site and Access Channel
}

\author{
Vincent Lafaquière, Sophie Barbe, Sophie Puech-Guenot, David Guieysse, \\ Juan Cortés, Pierre Monsan, Thierry Simeon, Isabelle André, Magali Remaud \\ Simeon
}

\section{To cite this version:}

Vincent Lafaquière, Sophie Barbe, Sophie Puech-Guenot, David Guieysse, Juan Cortés, et al.. Control of Lipase Enantioselectivity by Engineering the Substrate Binding Site and Access Channel. ChemBioChem, 2009, 10 (17), pp.2760-2771. 10.1002/cbic.200900439 . hal-01986285

\section{HAL Id: hal-01986285 \\ https://hal.laas.fr/hal-01986285}

Submitted on 18 Jan 2019

HAL is a multi-disciplinary open access archive for the deposit and dissemination of scientific research documents, whether they are published or not. The documents may come from teaching and research institutions in France or abroad, or from public or private research centers.
L'archive ouverte pluridisciplinaire HAL, est destinée au dépôt et à la diffusion de documents scientifiques de niveau recherche, publiés ou non, émanant des établissements d'enseignement et de recherche français ou étrangers, des laboratoires publics ou privés. 


\title{
Control of lipase enantioselectivity by engineering the substrate binding site and access channel
}

\author{
Vincent Lafaquière, ${ }^{[a]}$ Sophie Barbe, ${ }^{[a]}$ Sophie Puech-Guenot, ${ }^{[a]}$ David Guieysse ${ }^{[a]}$ \\ Juan Cortés, ${ }^{[c]}$ Pierre Monsan, ${ }^{[a, b]}$ Thierry Siméon, ${ }^{[c]}$ Isabelle Andrée ${ }^{[a]}$ and Magali \\ Remaud-Siméon ${ }^{*}[a]$
}

Lipase from Burkholderia cepacia (BCL) has proven to be a very useful biocatalyst for the resolution of 2-substituted racemic acid derivatives which are important chiral building blocks. Our previous work showed that enantioselectivity of the wild-type BCL could be improved by chemical engineering of the substrate molecular structure. From this earlier study, three amino acids (L17, V266 and L287) were proposed as targets for mutagenesis aiming at tailoring enzyme enantioselectivity. In the present work, a small library of 57 $B C L$ single mutants targeted on these three residues was constructed and screened for their enantioselectivity towards $(R, S)$-2-chloro ethyl

\section{Introduction}

A great challenge for pharmaceutical and industrial chemistry of the next decades is the discovery and development of fast and economic resolution processes. To obtain pure enantiomers, various methods such as diastereomeric salts, racemate resolution, or asymmetric synthesis using chiral auxiliaries and chiral catalysts can be used. ${ }^{[1-4]}$ Among the different approaches, biocatalysis based on the enzymatic resolution of racemic mixtures remains a method of choice. In particular, lipases (triacylglycerol hydrolases, EC 3.1.1.3) are among the most employed catalysts in organic synthesis to catalyze kinetic resolution of a wide range of substrates yielding optically pure compounds.

Recent advances in tailoring enzymes for high activity and selectivity and their combined use with chemo-catalytic reactions has expanded the role of biocatalysis to produce enantiopure compounds from racemic mixtures. Directed evolution or rational design techniques have proven to be successful to develop lipases with either enhanced or inverted enantioselectivity compared to the parental enzyme. ${ }^{[5-7]}$ Amongst lipases used for racemic resolution, lipase from Burkholderia cepacia (BCL) has proven to be very useful for the resolution of racemic mixtures of primary and secondary alcohols ${ }^{[8]}$ and carboxylic acids. ${ }^{[9,10]}$ In a previous study ${ }^{[11,12]}$, focused on the understanding of the factors involved in racemic resolution of 2-substituted carboxylic acids, it was suggested that given the topology of $B C L$ active site, which is located at the bottom of a narrow $17 \AA$ pocket, the enzyme ligand affinity and, by extension enantioselectivity, could be affected by 2-bromophenylacetate. This led to the fast isolation of three single mutants with a remarkable 10 times enhanced or reversed enantioselectivity. Analysis of substrate docking and trajectories in the active site was then performed. From this analysis, the construction of 13 double-mutants was proposed. Among them, an outstanding improved mutant of BCL was isolated that showed an E-value of 178 and a 15 times enhanced specific activity compared to the parental enzyme, thus demonstrating the efficiency of the semi-rational engineering strategy.

the accessibility of the substrate to the catalytic site and the difficulty encountered by the substrate in adopting a productive conformation at the reaction site.

In preliminary molecular modelling work $^{[11]}$, path-planning algorithms, originating from robotics and adapted for the investigation of various molecular motion problems ${ }^{[13-22]}$, were applied for investigating the access/exit of ligands to $B C L$ active site. The amino acids hindering the displacement of $(R, S)$ enantiomers along the active site pocket were highlighted using the atom distance information collected during the path searches by the collision detector $\mathrm{BioCD}^{[23]}$ integrated in the software prototype BioMove3D. ${ }^{[16,24]}$ On the basis of the analysis of contacts found along the computed access, two amino acids, L17 and V266, were identified as playing a major role in the discrimination of the pairs of enantiomers, and were proposed as targets for mutagenesis aiming at tailoring enzyme enantioselectivity.

In the present work, we have constructed libraries of variants targeted on amino acids identified by path analysis and located

[a] V. Lafaquière, Dr. S. Barbe, Dr D. Guieysse, Dr. S.Puech-Guenot, Prof. P. Monsan, Dr. I. André, Prof. M. Remaud-Siméon

Université de Toulouse; INSA, UPS, INP, LISBP, 135 Avenue de Rangueil, F-31077 Toulouse, France; CNRS, UMR5504, F-31400

Toulouse, France; INRA, UMR792 Ingénierie des Systèmes Biologiques et des Procédés, F-31400 Toulouse, France E-mail: magali.remaud@insa-toulouse.fr

[b] Prof. P. Monsan

Institut Universitaire de France, 103 boulevard Saint-Michel, F-75005 Paris

[c] Dr. J. Cortés, Dr. T. Siméon LAAS-CNRS, 7, avenue du Colonel-Roche, 31077 Toulouse, France 
both in the bottleneck and at the entrance of BCL active site. The libraries have been first screened on the basis of enzyme activity toward para-nitrophenyl butyrate ( $p$ NPB) substrate. Then, the enantioselectivity of the mutants towards $(R, S)$-2-chloro ethyl 2bromophenylacetate substrate (1) has been experimentally determined. The paper also discusses on the interpretation of molecular modelling results with regards to the understanding of $B C L$ enantioselectivity.

\section{Results and Discussion}

\section{Construction of the single mutant library}

Three amino acids were chosen as mutagenesis targets: L17, V266 and L287 (Figure 1). The selection of L17 and V266 was based on a previous study ${ }^{[11]}$ in which we showed that the bottleneck formed by these residues in BCL active site influences both the trajectory and the positioning of various two substitutedacids. The third amino acid, L287, was selected to examine the possible role of a residue located along the access channel but farther from the catalytic centre. Noteworthy, mutation of L287 residue was found in the V266L-L287I-F221L variant of $B$. cepacia lipase (Ps-FVL mutant from Amano CO., Japan), which displayed an enantioselectivity enhanced by 200 folds compared to the wildtype $\mathrm{BCL}$ for the resolution of $(R, S)$-2-bromophenyl acetic acid ethyl ester. ${ }^{[12]}$ To further investigate the role of these residues on enzyme enantioselectivity, the three amino acids (L17, V266 and L287) were systematically replaced by the 19 other possible amino acid residues to obtain a library of 57 single mutants.

Figure 1. Representation of B. cepacia lipase structure. A) Cross-section view of the enzyme active site. Catalytic triad residues (D264, H286, S87) are coloured in yellow. Amino acid residues forming the oxyanion hole (L17 and Q88) are coloured in cyan. The three amino acid residues selected as mutagenesis targets are: L17, V266 and L287. B) Overall fold of BCL is shown in a cartoon representation in which $\beta$-strands are represented by arrows and helices as coils. The region 1-117 is coloured in Purple, lid (118-159) in red, region 160-213 in grey, sub-domain facing the lid (214-261) in blue and region 262-320 in light brown. The position of a $\mathrm{Ca}^{2+}$ is indicated by a sphere. The catalytic triad (S87, D264 and H286) and amino acid residues involved in oxyanion hole stabilization (L17 and Q88) are shown in stick.

\section{Preliminary Screening on pNPB substrate}

The variants were first grown in deep-well plates. As shown in Figure 2, bacterial growth was rather homogenous for all of them. Following cell lysis, the mutants were screened for their ability to hydrolyze para-nitrophenyl butyrate ( $p N P B)$ (Scheme 1A), a substrate easily monitored by spectrophotometry at $405 \mathrm{~nm}$ and often used to determine lipase activity. ${ }^{[25]}$ The variability of $p$ NPB hydrolytic activity found amongst the variants is shown in Figure 2. Overall, 10 variants out of 57 appeared more active on $p$ NPB than the wild-type BCL. In particular, replacement of V266 by polar and uncharged Thr, Cys, Asn or GIn amino acids led to variants displaying a 2-4 folds enhanced hydrolytic activity, the highest enhancement being reached for V266N variant (Figure 2). In contrast, almost all the mutations at positions 17 and 287 had either a neutral or a negative effect by reducing the hydrolytic activity.

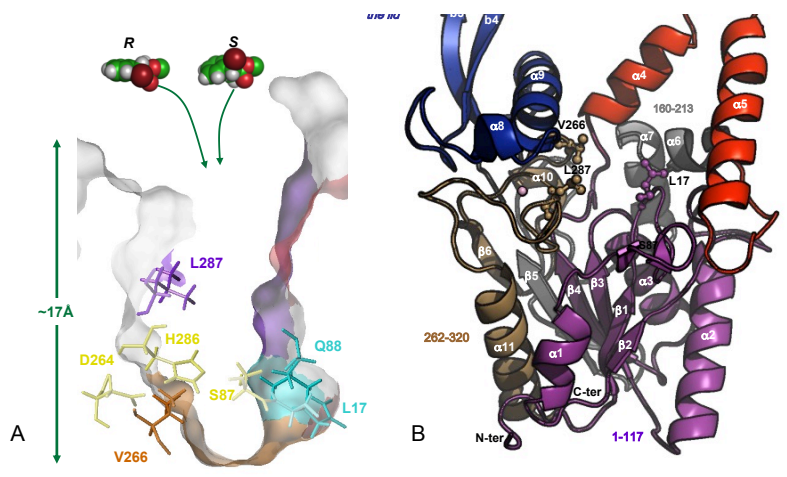

Scheme 1. Hydrolysis of A) para-nitrophenyl butyrate ( $p N P B)$ and $B)(R, S)-2$ -

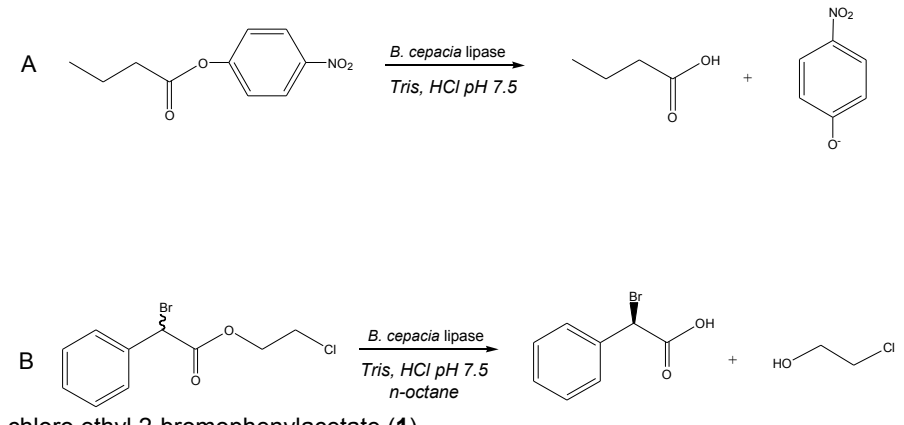

chloro ethyl 2-bromophenylacetate (1).

Figure 2. Profile of cell density and activity obtained for wild-type and variants of $\mathrm{BCL}$ expressed in E. coli JM109. Activity and OD600 nm were an average of

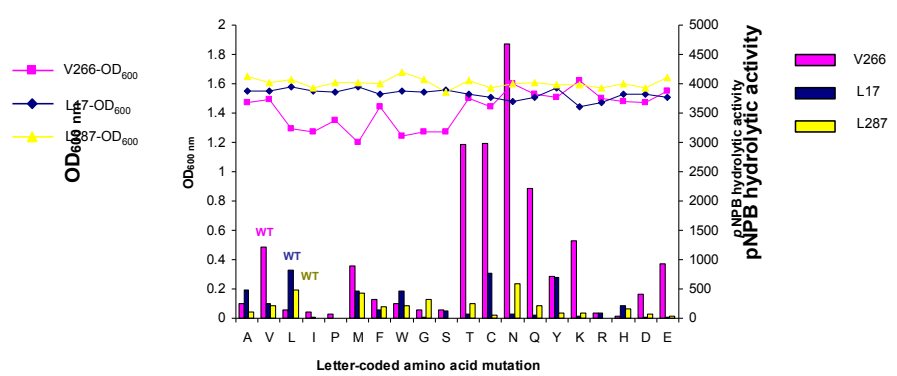

values obtained in 8 wells.

\section{Enantioselectivity of $\mathrm{BCL}$ single mutants towards $(R, S)-2$ - chloro ethyl 2-bromophenylacetate (1)}

The library of single mutants produced in 96-deep well plates was assayed in the presence of racemate 1 (Scheme 1B). Reactions to determine enantioselectivity were performed in deep-wells using rough extracts. The enantiomeric excess of the substrate (e.e.s) was plotted as a function of the conversion for all $\mathrm{BCL}$ variants (Figure 3). As shown in the figure, the single mutations at positions 266 and 287 had limited effect on enzyme enantioselectivity. In one single case, a variant was found to reverse its enantiopreference from the $(R)$ - form to the $(S)$ substrate. Indeed, when changing V266 by the most compact Glycine amino acid, also known to contribute greatly to conformational flexibility of polypeptide chains, the V266G variant was endowed with (S)-selectivity. Conversely, three L17 variants (namely L17S, L17G, and L17M) showed an enhanced enantiopreference for the $R$ enantiomer (Figure 3B). Variants L17S, L17G and L17M were produced from cultures in Erlenmeyer to validate the microtiter-plate assay. They exhibited $E$-values of 87, 24 and 78, respectively, showing that microtiter-plate assay was reliable to screen for enantioselectivity. 

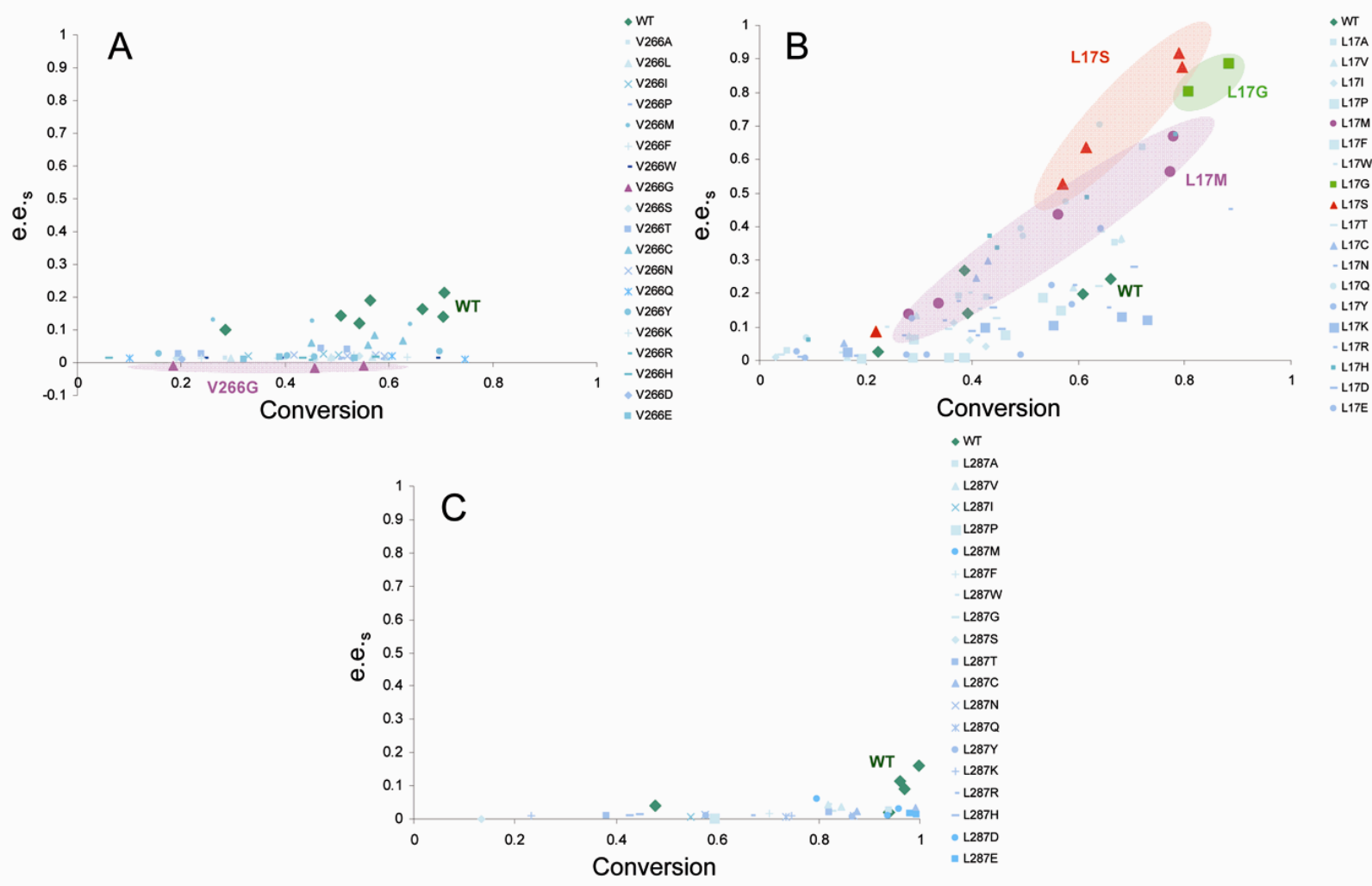

Figure 3. Evolution of substrate enantiomeric excess (e.e.s) as a function of conversion for the single variants on position V266 (A), L17 (B) and L287 (C). Enantiomeric excess was calculated as defined below: e.e. $._{\mathrm{s}}=\{[S]-[R]\}_{\mathrm{s}} /\{[S]+[R]\}_{\mathrm{s}}\left(\mathrm{s}=\right.$ substrate) and the conversion : c $=1-\left[(R+S)_{t} /(R+S)_{\mathrm{t}=0}\right]$. The results for the best variants are highlighted.

\section{Characterization of V266G, L17S and L17M variants}

The most enantioselective variant (L17S and L17M) and the one showing a reverse enantioselectivity (V266G) were then characterized in more details. Following production and purification by immuno-affinity, their ability to hydrolyze substrate 1 was determined. Regarding the V266G mutant, the specific activity remained unchanged compared to the wild-type enzyme (vi $=0.38 \mathrm{mU} / \mathrm{mg})$ but the reversal of enantioselectivity was confirmed when assaying the purified enzyme. The V266G variant was indeed found to hydrolyze the S-enantiomer significantly faster than the $R$ - one with an $E$-value of 20 (Table 1). Compared to the parental wild-type enzyme, variants L17S and L17M showed an increase of up to 6 folds in the initial rate of the $R$ enantiomer consumption (viR). In parallel, the initial rate of $S$ enantiomer consumption (viS) decreased by up to 3 folds (Table 1). This resulted in a 4 and 5 fold improvement of the specific activity of the L17S and L17M variants, respectively, compared to the wild-type enzyme. In similar way, the enantioselectivity of these mutants was increased by a remarkable 10 fold respect to wild-type BCL.

Insights on structural features controlling activity and selectivity of $\mathrm{BCL}$ single mutants through molecular modelling

Dynamics of the improved single mutants was first investigated to ensure that the mutations did not induce major modifications in the flexibility of the enzyme structure nor in the lid conformation of BCL variants. ${ }^{[26]}$ Dynamics studies were then completed by $i$ ) the analysis of the binding interactions of each enantiomer in the active site of the variants and ii) the analysis of substrate trajectories during the transport out of the active site.

Table 1. Specific hydrolytic activity and Enantioselectivity values determined for the wild-type BCL and its three single mutants towards each enantiomer of the racemic substrate $(R, S)$-2-chloro ethyl 2-bromophenylacetate (1).

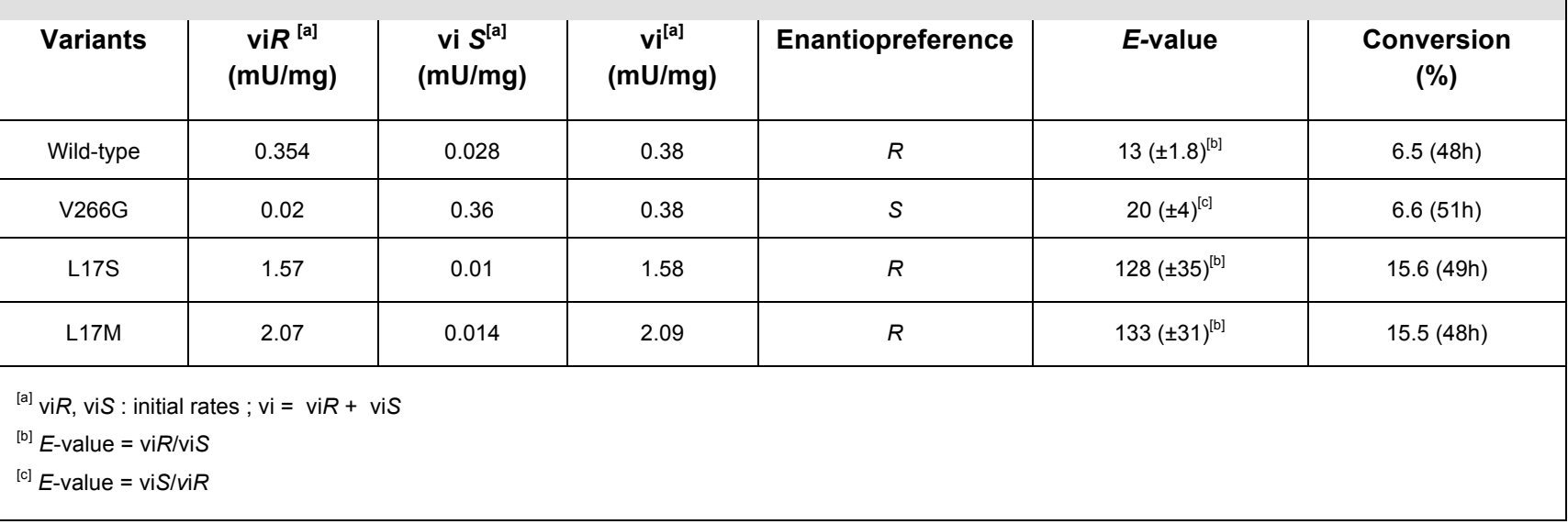


Dynamics of wild-type BCL and its variants at water/octane interface

The starting model of wild-type $B C L$ was built from the X-ray structure of $B C L$ in open conformation ${ }^{[27]}(\mathrm{PDB}: 3 \mathrm{LIP})$ which adopts the characteristic $\alpha / \beta$-hydrolase fold ${ }^{[28]}$ (Figure 1B). The model was then subjected to a 20 ns molecular dynamics (MD) simulation under constant temperature and pressure conditions in an explicit water/octane interface, in which the enzyme is known to be active, following a procedure previously described. ${ }^{[26]}$ In a similar way, we performed MD simulations on L17S, L17M and V266G mutants using 3D-models generated from the wild-type $\mathrm{BCL}$ model. To verify the stability of the MD trajectories, we inspected the potential energy of the system and the RMSD throughout the simulations. The RMSD trajectories calculated for the backbone atoms of the proteins were compared to the initial structures over the $20 \mathrm{~ns}$ of simulations. All profiles show a plateau with an average RMSD around $2 \AA$, reached after 2 ns, indicating that the systems have reasonably converged to stable states (Figure 4). To detect the regional motions in the protein structures, B-factors were calculated from MD simulations (Figure $5 A$ ). Main differences between the wild-type $B C L$ and its variants reside in two regions, mostly constituted by hydrophobic amino acid residues, surrounding the catalytic pocket. The first region is defined by the lid (residues 118-159) while the second (residues 221-246) corresponds to a sub-domain facing the lid (Figure 1B). Simulated B-factors revealed a particularly high mobility of the hydrophobic $\beta$-hairpin constituted by the b3 and b4 strands for all variants as well as for the wild-type BCL. Of note, the mobility of the loop between the $\alpha 5$ and $\alpha 6$ helices was found more pronounced for the $\mathrm{V} 266 \mathrm{G}$ variant than for the wild-type $\mathrm{BCL}$ (Figure 5A).

Comparison of initial structures with those obtained after 20 ns MD simulation shows that wild-type BCL and its mutants stay in the wide open conformation observed in the crystal (Figure 5B-E). Overall, no major effect of the mutation on the flexibility of the protein backbone was observed. The two variants exhibited similar conformational rearrangements along the simulation and residues involved in the catalytic machinery located at the bottom of a narrow and deep pocket (catalytic triad: S87, H286, D264 and oxyanion hole stabilisation: Q88 and L17) did not undergo any significant variation. These results suggest that the amino acid substitutions introduced in BCL did not perturb the protein folding.

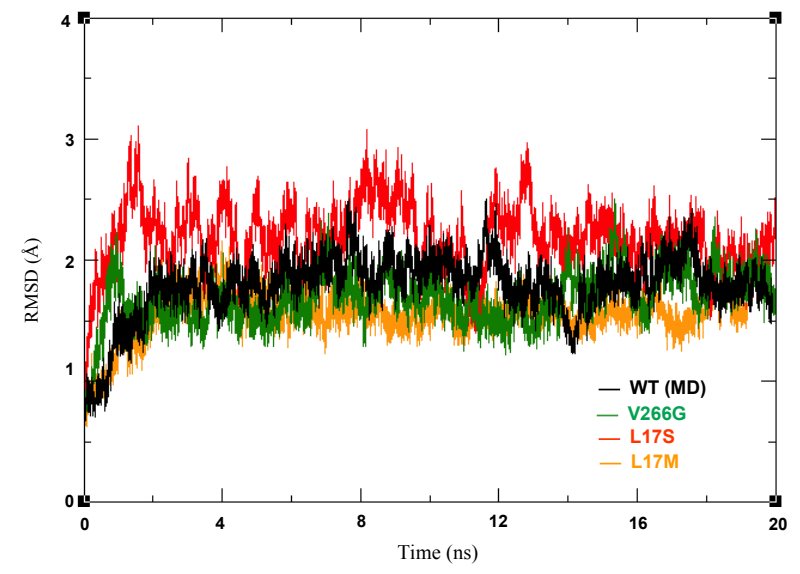

Figure 4. Time variation of the RMSD of backbone atoms of the proteins (wildtype (black), V266G (green), L17S (red) and L17M (orange) variants) during the course of MD simulations carried out in explicit water/octane solvent.
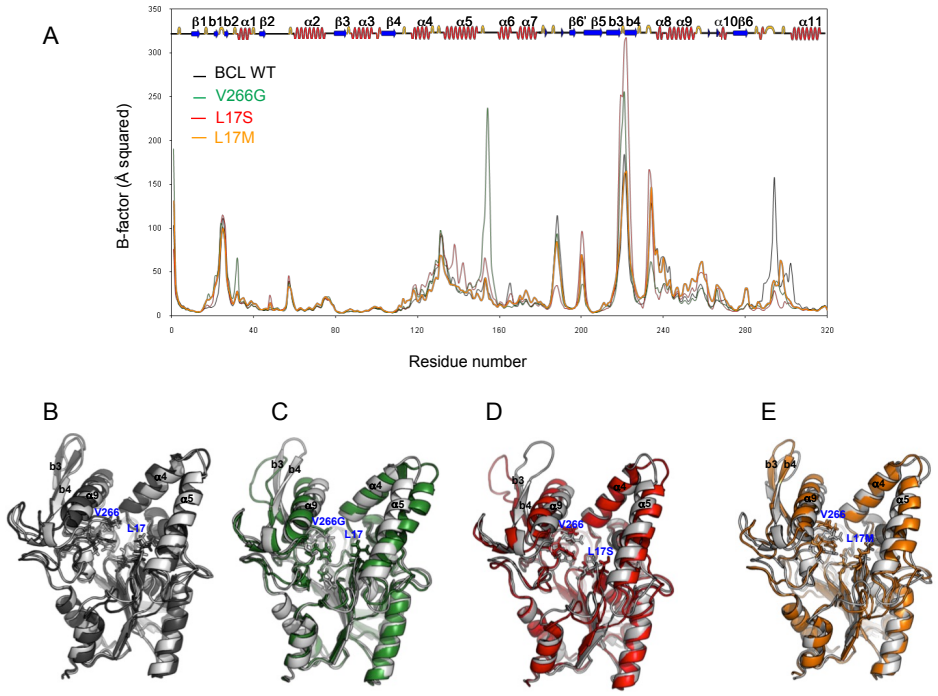

Figure 5. A) Calculated B-factors of $B C L$ residues from $M D$ simulations carried out in an explicit water/octane interface for wild type BCL (black), for variant V266G (green), for L17S (red) and for L17M (orange). The secondary structure of $B C L$ is shown on the graph for reference. Snapshots of the enzyme backbone structures taken at the beginning of the production phase (grey coloured) and after $20 \mathrm{~ns}$ of $\mathrm{MD}$ simulation in water/octane environment: B) wild-type $\mathrm{BCL}$ (dark grey), C) V266G (green) D) L17S (red) and E) L17M (orange).

Covalent docking of substrate in active sites of wild-type BCL, L17S, L17M and V266G variants

Using a covalent docking procedure, we generated models of the substrate covalently bound to each enzyme, which corresponds to the tetrahedral intermediate known to be a good mimic of the transition state. In wild-type BCL, L17S and L17M variants, the bulky bromine atom of the rapidly transformed $R$-enantiomer is positioned in the inner hydrophobic pocket, the so-called acyl pocket $\mathrm{HA}^{[12]}$, pointing toward the inner $\mathrm{L} 167$ with the aromatic ring pointing towards the outside of the catalytic pocket (Figure 6A). In contrast, the least rapidly hydrolyzed $S$-enantiomer was also docked with its bromine atom in the HA pocket, but oriented toward the bottleneck-forming V266. Interestingly, mutation L17S also introduced an additional hydrogen bonding interaction with the covalent intermediate, leading to a greater stabilization of the transition states for both $R$ - and $S$ - enantiomers compared to the wild-type BCL (Figure 6C). These results are in agreement with a greater hydrolytic activity of L17S variant compared to the wildtype BCL toward substrate 1 (Table 1). However, the difference between scores of the $S$ - and $R$ - enantiomers in L17S is lower than that observed for the wild-type enzyme, thus failing to explain the 10 fold increase of enantioselectivity observed for the variant in favour of the $R$-enantiomer. The docking scores obtained respectively for the $R$ - and the $S$ - enantiomers in the L17M variant are in agreement with the enantiopreference of the enzyme. Regarding V266G variant, the mutation uncluttered the active site and thus favoured the docking of the $S$-enantiomer of substrate 1 in the catalytic site, as reflected by the more favourable docking score (Figure 6B). Figure 6 shows that the orientation of the aromatic ring in the docked poses within all enzymes varies between the $R$ - and the S-enantiomers, except for the V266G variant. In the latter, the aromatic ring fits nicely in the space left empty by the V266G mutation, allowing a stacking of the aromatic ring onto the catalytic $\mathrm{H} 286$. Close values of docking scores were obtained for both enantiomers ie -1.03 and -1.20 for the $R$ - and $S$ enantiomers, respectively. The favourable docking score for the $S$ enantiomer is in agreement with the experimentally observed reverse of enantiopreference exhibited by the V266G variant. 
A

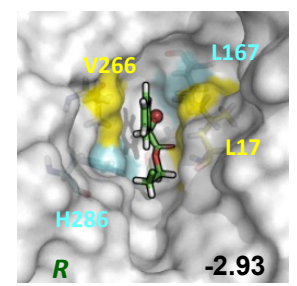

B

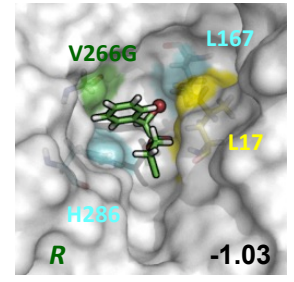

C

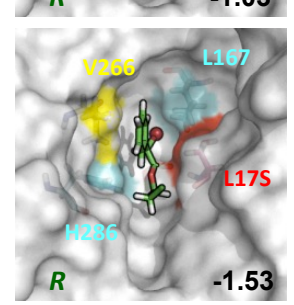

D

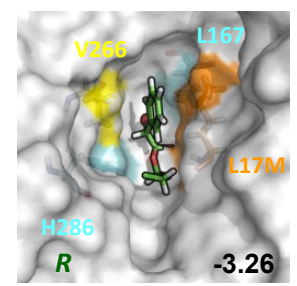

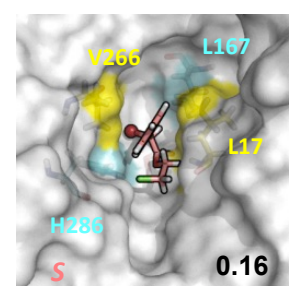
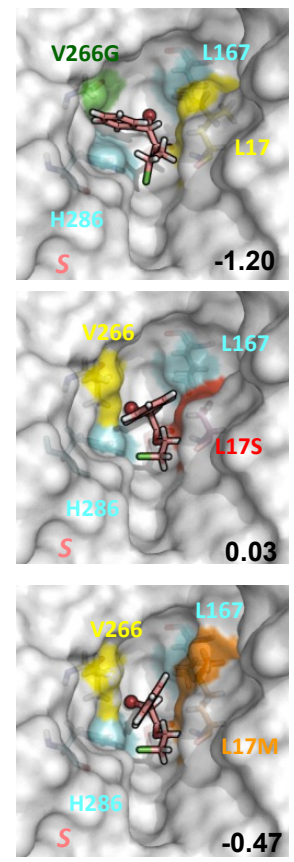

Figure 6. Highest score docking modes of $(R, S)$-2-chloro ethyl 2bromophenylacetate (1) covalently bound in the enzyme active site. 1 is docked in the catalytic pocket of wild type enzyme is represented on A) in V266G variant on B), in L17S on C) and in L17M on D). Bromine atom is shown as a sphere. Docking scores are indicated on the figures.

Overall, the docking scores (Figure 6) are in agreement with the enantiopreference but they failed to predict quantitatively the enantioselectivity, in particular the large improvement of L17S and L17M enantioselectivity. Molecular docking was performed on rigid enzyme 3D-models and solely, the substrate was allowed to adapt its conformation to the active site. Given that molecular adaptation and flexibility are important components for an accurate description of specific ligand binding interactions, this is obviously a limitation.

Computing substrate pathways to BCL single mutants catalytic site

Dynamic simulations indicate that the amino acid substitutions introduced in $B$. cepacia lipase did not perturb the protein folding. However, they contribute to significant changes in the enantiomer accessible volume and may have an impact on substrate accessibility. The geometrically feasible motions of the substrates within the enzyme active site were explored using the pathplanner integrated in the Biomove3D software. ${ }^{[16,24]}$ The algorithm used in this work is derived from the previously described disassembly path-planning technique of articulated objects. ${ }^{[11,24,29]}$ In the new version of the Biomove3D software, the basic RRT algorithm was replaced by ML-RRT (Manhattan-Like Rapidlyexploring Random Tree) algorithm. ${ }^{[24]}$ ML-RRT outperforms the

basic RRT algorithm by decreasing considerably the computing time and its variance. Furthermore, it is able to efficiently treat proteins models with all side chain being potentially flexible. ${ }^{[24]}$ Within a few minutes on a standard mono-processor PC, the algorithm is able to compute ligand pathways while taking into account the entire flexibility of both the ligand and the protein sidechains that are required to move during the computation of the pathways. In the latest version of Biomove3D, a new computational tool has been included that facilitates the analysis of the ML-RRT search trees by encoding molecular motions in voxel maps. ${ }^{[30]}$ Such representation permits to arrange the information obtained from the exploration of a high-dimensional space (the conformational space of the molecular model) into a three-dimensional data structure. By combining a geometric conformational exploration and an arrangement of the resultant information into a voxel map, the method permits to visualize the differences between the space explored by the different enantiomers when accessing the catalytic site of the different $\mathrm{BCL}$ enzymes. Starting from the docked tetrahedral intermediate of the $R$ - and $S$ - enantiomer of substrate 1 , we sampled the space that the enantiomers can explore to go from the bottom (catalytic site) of selected BCL variants (L17S, L17M and V266G) to the protein surface. The voxel-maps reflect the volume of the catalytic pocket explored by the substrate during its access/exit pathways to the active site. In Figure 7 are shown the computed voxel maps which represent the positions that can be geometrically reached by the substrate center of mass during the conformational explorations. As voxels have been coloured following the chronological order of generation, one can easily identify the regions that the substrate reaches first during the geometrical exploration. Comparison of the voxel-maps reveals significant differences in the behaviour of racemate 1 into the different enzyme catalytic pockets. Voxelmaps obtained for the wild-type BCL indicate a narrower distribution for the $S$ - enantiomer than the $R$ - form, reflecting a more constrained motion of the $S$-enantiomer when going through the bottleneck formed by V266 and L17. Light-blue voxels are found from the bottom to the entrance of the catalytic pocket for the $R$-enantiomer indicating a faster displacement requiring less iterations than for the $S$-enantiomer for which orange voxels are seen around the bottleneck revealing a geometrically difficult passage. These results are in agreement with the faster conversion of the $R$-enantiomer by the wild-type $\mathrm{BCL}$ (Table 1). As can be seen by the larger voxel map, the introduction of the V266G mutation notably facilitates the movement of the $S$ enantiomer in the mutant active site compared to the parental enzyme. On the other hand, the V266G mutation exposed a secondary pocket (encircled in Figure 7B) that is unsuccessfully explored by the $R$-enantiomer when searching a way out of the pocket. The exploration of this dead-end region resulted in an increase of the CPU time taken by the path planner to find a solution. Interestingly, upon L17S mutation, the topology of the active site favoured considerably the movement of the $R$ enantiomer along the access/exit pathway. Indeed, a network of dark-blue voxels connects the bottom to the top of the catalytic site revealing the existence of pathways requiring less iterations than for the wild-type BCL to exit the catalytic pocket. Of note, the voxel maps computed for the S-enantiomer in the wild-type and the L17S variant are nearly the same, what is in agreement with the comparable initial rates determined experimentally. Overall, the computation of the exit pathway is greatly facilitated for the $R$ enantiomer upon the L17S mutation, what is in qualitative agreement with the 10-fold enhanced enantioselectivity of the L17S variant compared to the wild-type enzyme. The differences observed between the $R$ - and the $S$ - voxel maps in the L17M 
variant reflect the variations of the behaviour of each of the enantiomer during its displacement along the active site. Indeed, the explored space of the $S$-enantiomer is significantly reduced compared to the R-form, indicating thus a greater difficulty of the active site exploration to find an exit pathway. These differences are in agreement with the $E$-value of 133 in favour of the $R$ substrate.

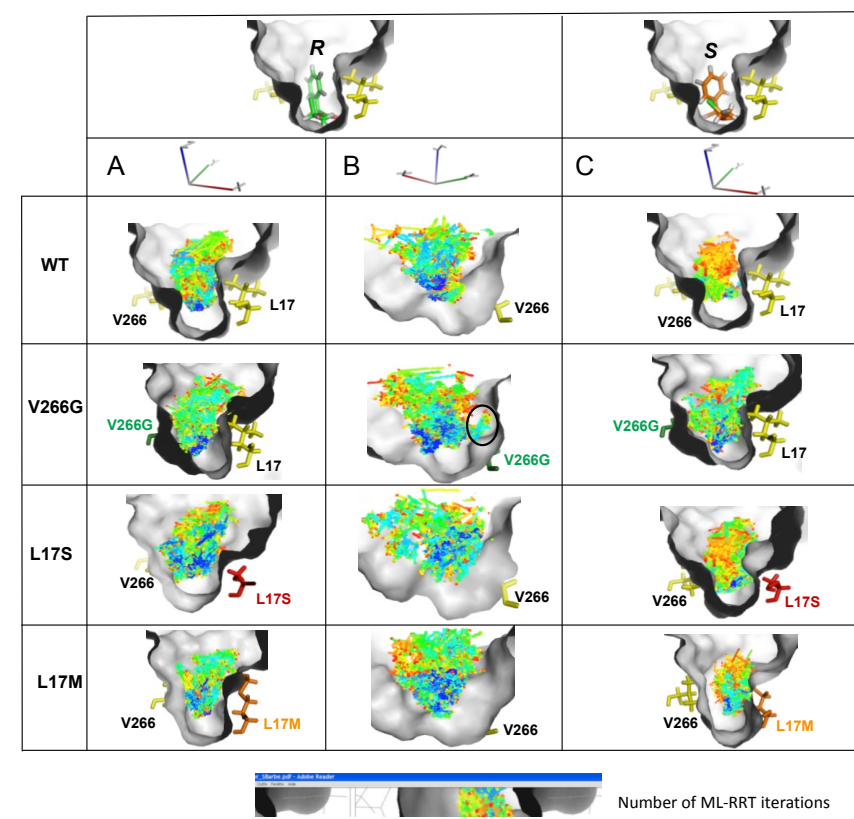

Figure 7. Voxel maps representing locations of the center of mass of the $(R, S)$ enantiomers of $(R, S)$-2-chloro ethyl 2-bromophenylacetate (1) reachable from the catalytic position within the wild-type BCL, V266G, L17S and L17M mutants. Voxels resolution is $0.1 \AA$ and colours indicate the chronological order of generation.

In the examples presented here, it clearly appears that modelling of the tetrahedral intermediates for both enantiomers was not enough to explain the differences observed in the enantioselectivity of $\mathrm{BCL}$ and its variants. Often neglected, the effect of entropy variation has been shown in many cases to be as important as the enthalpic component in enzyme enantioselectivity. ${ }^{[31,32]}$ Several studies have attempted to understand the role of entropy on a molecular level and they have estimated the substrate accessible volume within the active site and showed that it could be correlated to transition state entropy. ${ }^{[31,32]}$ The modelled volumes could in some cases predict the correct enantiopreference and the topology of the active site is also proposed to be more suited for the near-attack conformation of the favoured enantiomer. ${ }^{[33]}$ In our approach, the full flexibility of both the protein side chains and the substrate is considered for the calculation of the enantiomer pathways. In view of the importance of taking into account the entire molecular motions involved in catalysis when modelling the enzyme reaction, we believe that the computational methods applied in this work might help to expand our knowledge on how enzymes distinguish between enantiomers and to understand the role of entropy on a molecular level.

\section{Designing improved BCL double-mutants}

The enantiomers docking, access trajectories and voxel map analyses confirmed the predominant role of the V266-L17 bottleneck on racemate 1 accommodation. To generate diversity at these positions, we first selected the best single mutants L17S and L17M and attempted the introduction of an additional mutation at position V266 (Table 2). In the same way, we also attempted the modification of the substrate access to the active site by introducing an additional mutation at position 287 in mutant L17S. Docking scores, analysis of collision and voxel maps were used to target the mutations. A total of 13 double mutants were constructed and their enantioselectivity was roughly determined without purification of the variants. As shown in table 2, except for L17M/V266M mutant, none of the double mutants targeted at positions 17 and 266 led to a notable improvement of the enzyme enantioselectivity. It underlines that engineering of the active site bottleneck is a tricky task, likely due to the proximity of the catalytic triad and oxyanion hole. The behaviour of the L17S/L287 double mutants turned out to be quite interesting as all of them were found more enantioselective than the wild type BCL. Altogether, four mutants were finally purified to homogeneity and characterized. As shown in Table 3, L17M/V266M enantioselectivity was confirmed. The mutant was found slightly more enantioselective than the single L17M mutant and also slightly more active. Regarding, the double mutants L17S/L287A; L17S/L287I and L17S/L287W, an interesting modulation of the enantioselectivity toward racemate 1 was observed that correlates pretty well with the steric hindrance of the substituent at position 287. In addition, these results show that distant mutations allow a

\begin{tabular}{|c|c|}
\hline Variants & $E$-value ${ }^{[a]}$ \\
\hline Wild-type & 12 \\
\hline \multicolumn{2}{|c|}{ Bottleneck: L17S-V266 combinations } \\
\hline Single mutant : L17S & 87 \\
\hline L17S/V266I & 19 \\
\hline L17S/V266M & 15 \\
\hline L17S/V266Q & 1 \\
\hline L17S/V266F & 18 \\
\hline L17S/V266D & 2 \\
\hline L17S/V266W & 1 \\
\hline L17S/V266L & 4 \\
\hline L17S/V266T & 10 \\
\hline \multicolumn{2}{|c|}{ Bottleneck: L17M-V266 combinations } \\
\hline Single mutant : L17M & 78 \\
\hline L17M/V266F & 9 \\
\hline L17M/V266M & 122 \\
\hline \multicolumn{2}{|l|}{ L17S-L287 combinations } \\
\hline L17S/L287A & 39 \\
\hline L17S/L287I & 45 \\
\hline L17S/L287W & 24 \\
\hline
\end{tabular}


Table 3. Specific hydrolytic activity and Enantioselectivity values determined for the wild-type BCL and its six double-mutants towards each enantiomer of the racemic substrate $(R, S)$-2-chloro ethyl 2-bromophenylacetate (1). (Error range for the double-mutants is within 10 to $20 \%$ )

\begin{tabular}{|c|c|c|c|c|c|c|}
\hline Variants & $\begin{array}{c}\mathrm{vi} R^{[\mathrm{a}]} \\
(\mathrm{mU} / \mathrm{mg})\end{array}$ & $\begin{array}{l}\mathrm{viS}^{[\mathrm{a}]} \\
(\mathrm{mU} / \mathrm{mg})\end{array}$ & $\begin{array}{c}\mathrm{vi}^{[\mathrm{a}]} \\
(\mathrm{mU} / \mathrm{mg})\end{array}$ & Enantiopreference & $E$-value ${ }^{[b]}$ & $\begin{array}{c}\text { Conversion } \\
(\%)\end{array}$ \\
\hline Wild-type & 0.354 & 0.028 & 0.38 & $R$ & 13 & $6.5(48 h)$ \\
\hline L17M/V266M & 2.81 & 0.017 & 2.83 & $R$ & 166 & 9 (19h) \\
\hline L17S/L287A & 1.83 & 0.081 & 1.91 & $R$ & 22.5 & $15.6(20 \mathrm{~h})$ \\
\hline L17S/L287I & 5.95 & 0.033 & 5.98 & $R$ & 178 & $15.5(20 \mathrm{~h})$ \\
\hline L17S/L287W & 0.55 & 0.01 & 0.56 & $R$ & 55 & $6(20 h)$ \\
\hline \multicolumn{7}{|c|}{$\begin{array}{l}\text { [a] } \text { vi } R \text {, viS, vi : initial rates } ; \mathrm{vi}=\text { vi } R+\text { viS } \\
{\left[{ }^{[b]} E \text {-value }=\text { vi } R / \text { vi } S\right.}\end{array}$} \\
\hline
\end{tabular}

more subtle tune-up of the enzyme structure to enhance its enantioselectivity than mutations located near the catalytic center. With a remarkable $E$-value of 178 , the L17S/L287I variant was the most enantioselective BCL mutant isolated. Furthermore, this mutant was also found to be 15 times more active than the wildtype enzyme.

\section{Conclusion}

Overall, our study shows that the catalytic properties of $B$. cepacia lipase can be exploited "à la carte" for the kinetic resolution of 2substituted racemic acids by structural perturbations via amino acid substitution. The screening of a small library of only 57 monomutants on two structurally different substrates clearly revealed the importance of designing screening assays specifically adapted to the substrate of interest. Another achievement is that the screening of this library enabled the fast isolation of several lipases with a remarkable 10 times enhanced or reversed enantioselectivity for the resolution of $(R, S)$-2-chloro ethyl 2bromophenylacetate.

This work also shows the interest of using a semi-rational engineering strategy for the construction of mutants. The strategy we have developed, which combines classical and novel molecular modelling tools enabled us to select a restricted number of double mutants (only 13) to construct. Out of this library, the best isolated variant displayed a 15 fold increased activity and a 10 times enhanced enantioselectivity. Although additional analyses are now necessary to give a comprehensive interpretation of the molecular factors involved in those enhancements, we can still conclude on the basis of the positive hit ratio (15\% of enhanced variants out of the screened library) that the followed semi-rational engineering strategy was fairly efficient.

The computational analysis revealed differences in the interaction so the enzyme with the different enantiomers that could be linked to accessible volumes (represented using the voxel-map approach) and by extension to entropy variations. To accurately investigate the role of both enthalpy and entropy contributions on the enantioselectivity of the $\mathrm{BCL}$ variants, we would need to determine experimentally these thermodynamic parameters, which is currently unavailable (???). Nevertheless, our aim is to continue the development of novel computational methods for understanding enzyme enantioselectivity.

\section{Experimental Section}

Site directed mutagenesis: Using the plasmid pFLAG-ATS-Lip-Hp as a template, the QuickChange Site-Directed Mutagenesis Kit (Stratagene, La Jolla, CA) was used according to the manufacturer's instruction to introduce 19 other amino acids at positions $266^{[25]}, 17$ and 287. The primers employed are L17_forw (5'-CTC-GTG-CACGGG-XXX-ACG-GGC-ACC-GAC-3') and L17_rev (5'-GTC-GGT-GCCCGT-XXX-CCC-GTG-CAC-GAG-3'), where $\bar{X}$ corresponds to the 19 other amino acids (L17A_forw=GCC; L17A_rev=GGC; L17C_forw=TGC; L17C_rev=GCA; L17D_forw=GAC; L17D_rev=GCT;

L17F forw=TTC; L17F rev=GAA; L17G forw=GGC; L17G rev=GCC; L17H_forw=CAC; L17-_H_rev=GTG; L17I_forw=ATC; L17I_rev=GAT; L17M_forw=ATG; L17M_rev=CAT; L17N_forw=AAC; L17N_rev=GTT; L17P_forw=CCC, $\quad$ L17P_rev=GGG; $\quad$ L17Q_forw=CAG; L17Q_rev=CTG; $\quad$ L17R_forw=CGC; $\quad$ L17R_rev=GCG: L17S_forw=AGC; L17S_rev=GCT; L17T_forw=ACC; L17T_rev=GGT; L17V forw=GTC; $\quad$ L17V rev=GAC; $\quad$ L17W forw=TGG; L17W_rev=CCA; L17Y_forw=TAC; L17Y_rev=GTA; L17E_forw=GAG; L17E_rev=CTC; L17K_forw=AAG; L17K_rev=CTT).

The primers employed for position L287 are L287_forw (5'-CG-AGCTAC-AAG-TGG-AAC-CAT-XXX-GAC-GAG-ATC-AAC-CAG-3') and L287_rev (5'-CTG-GTT-GAT-CTC-GTC-XXX-ATG-GTT-CCA-CTTGTA-GCT-CG-3') where XXX correspond to the codon of the other 19 amino acids (L287A_forw=GCC, L287A_rev=GGC; L287C_forw=TGC, L287C_rev=GCA; L287D_forw $=\overline{G A C}, \quad$ L287D_rev $=G T C$; L287E_forw=GAG, L287E_rev=CTC; $\quad$ L287F_forw $=T T C$, L287F_rev=GAA; L287G_forw=GGC, L287H_forw=CAC, L287L287I_rev=GAT; $\quad$ L287K_forw=AAG, L287M_forw=ATG, $\quad$ L287M_rev=CAT; L287N_rev=GTT; $\quad$ L287P_forw $=C C C$, L287Q_forw=CAG, L287Q_rev=CTG; L287R_rev=GCG; L287S_forw=AGC L287T_forw=ACC, $\quad$ L287T_rev=GGT; L287V_rev=GAC; $\quad$ L287W_forw=TGG, L287Y_forw=TAC, L287Y_rev=GTA).

L287G rev=GCC L287!_forw=ATC, L287K_rev=CTT; $L 287 \mathrm{~N}$ forw $=A A C$, L287P rev=GGG; L287R_forw=CGC, L287S rev=GCT; L287V forw=GTC, L287W_rev=CCA;

Double mutants were constructed following the same procedure used for single mutants construction. Mutant plasmids were used as 
template for site directed mutagenesis (QuickChange Site-Directed Mutagenesis Kit). Primers employed are the same as described above.

Mutant plasmids were transformed into E. coli JM109 (Promega, Madison, $\mathrm{WI})$ ). Clones were stored in $15 \%$ glycerol $(\mathrm{v} / \mathrm{v})$ in cryotube vials (Nunc ${ }^{\mathrm{TM}}$ Brand Products, Denmark) at $-20^{\circ} \mathrm{C}$ and they were used to inoculate culture in 96-deep-well microplate (Nunc ${ }^{\mathrm{TM} B r a n d}$ Products, Denmark).

Production of BCL variant in microplate scale: Starter cultures in sterile 96-well microplates filled with $2 x$ YT medium $(150 \mu \mathrm{l})$ were inoculated using clones of wild-type or $\mathrm{BCL}$ variants (10 microliters) stored in cryotube. After $24 \mathrm{~h}$ of growth at $30^{\circ} \mathrm{C}$ under horizontal shaking $(250 \mathrm{rpm})$, starter cultures $(100 \mu \mathrm{L})$ were used to inoculate sterile 96-deep-wells culture for protein production (ABgene, UK; 1.1 $\mathrm{mL} 2 \mathrm{x}$ YT ; isopropyl- $\beta$-D-thiogalactopyranoside (IPTG) at $0.01 \mathrm{mM}$ supplied by Euromedex (France)).

Theses cultures were grown at $30^{\circ} \mathrm{C}$ during $24 \mathrm{~h}$ under agitation. Growth was measured by $\mathrm{OD}_{600 \mathrm{~nm}}$ after shaking to avoid settlement and the cells were then centrifuged at $3700 \mathrm{rpm}$ for $30 \mathrm{~min}$ at $4{ }^{\circ} \mathrm{C}$. The pellets were resuspended in lysis buffer $(200 \mu \mathrm{L} ; 100 \mathrm{mM}$ Tris- $\mathrm{HCl}$ $\mathrm{pH} 7.5$, protease inhibitor cocktail tablet EDTA free (Roche Diagnostic, France), $1 \mathrm{mg} / \mathrm{mL}^{-1}$ lysozyme (Euromedex), $5 \mu \mathrm{gg} / \mathrm{mL}^{-1}$ DNasel (Euromedex) and 1X BugBuster (Novagen, Germany), sonicated in a sonicating bath (4 cycles; 1 cycle: 2 min of sonication and 2 min on ice), and frozen at $-80^{\circ} \mathrm{C}$ over night. After thawing, crude extract were used to measure lipase activity and for screening procedures.

Determination of hydrolytic activity on pNPB of BCL variants produced in microplate: Clones were screened for their hydrolytic activity using an integrated robotic TECAN Genesis RSP-200 platform on para-nitrophenyl butyrate supplied by Sigma-Aldrich ( $p$ NPB) as substrate. Twenty microliters of the lipase extract diluted $(\mathrm{v} / \mathrm{v})$ in Tris$\mathrm{HCl}$ buffer $(100 \mathrm{mM}, \mathrm{pH}=7.5)$ were mixed with Tris-HCl buffer $(175$ $\mu \mathrm{L} ; 100 \mathrm{mM}, \mathrm{pH}=7.5)$ and $p N P B(5 \mu \mathrm{L} ; 40 \mathrm{mM}$ in solution in 2-methyl2-butanol). pNPB consumption was measured at $405 \mathrm{~nm}$ on a VersaMax tunable microplate reader (Molecular Devices, Sunnyvale, $\mathrm{CA}$ ) at $30{ }^{\circ} \mathrm{C}$. One unit was defined as the amount of enzyme that released $1 \mu \mathrm{mol}$ of $p$-nitrophenol per minute under these conditions. Activity measurements were performed once for each well. BCL is almost exclusively produced as inclusion bodies and only a small part of the enzyme is in the soluble fraction. The quantity of enzyme in soluble fraction varies between productions and the hydrolytic activity measured can thus be different. To overcome this problem, wild-type enzyme was produced in each deep-well plate and the hydrolytic activity of single variants was always compared to the wild-type enzyme of the same plate.

The activity values obtained from deep-well cultures and Erlenmeyer flask cultures were compared. A positive correlation was found between the hydrolytic activities produced either by erlenmeyer or deep-well cultures (data not shown), in agreement with that previously reported for V266 variants. ${ }^{[25]}$

Determination of $\mathrm{BCL}$ variants enantioselectivity toward hydrolysis of $(R, S)$-2-chloro ethyl 2-bromophenylacetate:

Classical test: When BCL variant were produced in erlenmeyer flasks using 2x YT medium (tryptone $16 \mathrm{~g} / \mathrm{L}$, yeast extract $10 \mathrm{~g} / \mathrm{L}, \mathrm{NaCl} 5 \mathrm{~g} / \mathrm{L}$ ) supplemented with ampicillin (100 $\mu \mathrm{g}$ per millilitre) using conditions previously reported ${ }^{[25]}$, a classical enantioselectivity test was used. Five hundred microliters of crude extract containing recombinant $\mathrm{BCL}$ was added to racemic substrate $(250 \mu \mathrm{L} ; 50 \mathrm{mM}$ in $n$-octane). The hydrolysis reaction was carried out at $30^{\circ} \mathrm{C}$ under magnetic stirring. The progress of the reaction was followed by sampling the reaction at regular intervals.

Miniaturized test: For this test, hydrolysis reaction was carried out in 96-deep-well plates used for the enzyme production. After lipase hydrolytic activity measurement, $(R, S)$-2-chloro ethyl 2bromophenylacetate $(100 \mu \mathrm{L} ; 50 \mathrm{mM}$ in $\mathrm{n}$-octane) were added into each well containing enzyme extract $(180 \mu \mathrm{L})$. The deep-well plate was sealed with a thermosealing system (Thermofischer scientific, ALPS $50 \mathrm{~V}$ ) and the reactions were shaken at $800 \mathrm{rpm}$ and $30^{\circ} \mathrm{C}$ in Infors Microtron. The reaction was stopped by addition of $n$ hexane/IPA ( $500 \mu \mathrm{L} ; 70 / 30 ; \mathrm{v} / \mathrm{v})$ and the reaction medium was mixed, centrifuged at $13000 \mathrm{rpm}$ during $5 \mathrm{~min}$. The organic extract was analysed by chiral liquid chromatography

HPLC analysis: The chiral HPLC instrument was equipped with a chiral column [Chiralcel OJ-H $(25 \mathrm{~cm} \times 4.6 \mathrm{~mm})$, Daicel Chemical Industries Ltd, Japan] connected to a UV detector (at $254 \mathrm{~nm}$ ). The following conditions were used: $n$-hexane/isopropanol 80:20, v/v ; flow rate of $1.0 \mathrm{~mL} \cdot \mathrm{min}^{-1}$ at $40^{\circ} \mathrm{C}$

Determination of conversions, substrate enantiomeric excess (e.e.s) and enantioselectivities ( $E$ values): The conversion was calculated from HPLC results using the following relationship: $\mathrm{C}=1$ $\left[(R+S)_{t}(R+S)_{t 0}\right]^{*} 100$. The substrate enantiomeric excess is calculated as: e.e.s $=\{[R]-[S]\}_{\mathrm{s}} /\{[R]+[S]\}_{\mathrm{s}}$ (s=substrate). Enantioselectivity value was determined as the ratio of the initial rate of $(R)$-enantiomer production versus the intial rate of $(S)$-enantiomer production : Evalue $=(\mathrm{vi} R / \mathrm{viS})$. Initial rates were determined, before $10 \%$ of substrate conversion, by linear regression over at least five points. For reaction on non-purified enzyme, E-value was calculated using the conversion, the substrate enantiomeric excess and the mathematical expression: $E$-value $=(\operatorname{Ln}[(1-C)(1-e . e . s)]) /(\operatorname{Ln}[(1-C)(1+$ e.e.s $)])$.

Purification and protein assay: Enzyme was expressed in E. coli JM109 in fusion with a FLAG-peptide. This peptide was used then to purify the enzyme by immuno-affinity purification using ANTI-FLAG M2 affinity gel (Sigma). Purification was made in batch. One volume of enzyme extract interacts with one volume of affinity gel during $1 \mathrm{~h}$ at $4^{\circ} \mathrm{C}$. One volume of gel was then washed by 36 volumes of TBS $(50$ $\mathrm{mM}$ TRIS, $150 \mathrm{mM} \mathrm{NaCl}, \mathrm{pH}=7.4$ ). Elution was made by interaction between gel and FLAG-peptide (Sigma) solved in TBS added $1 \%$ of triton X-100 (Euromedex) and $1 \%$ of Tween 20 (Sigma-Aldrich) at 300 $\mu \mathrm{g} / \mathrm{mL}$. One volume of gel interacts with one volume of elution solution during $5 \mathrm{~min}$. This step was performed three times. The assay of purified protein was made with a NanoDrop 1000 with $2 \mu \mathrm{L}$ of solution.

General procedure for the preparation of $(R, S)$-2-chloro ethyl 2bromophenylacetate (1): Compound 1 was synthesized according to the procedure previously described ${ }^{[34]}$, but using 2-chloro ethanol as alcohol.

Spectroscopic data: Infrared spectra were recorded on a Perkin Elmer, 1310 infrared spectrophotometer. ${ }^{1} \mathrm{H}$ and ${ }^{13} \mathrm{C}$ NMR spectra were recorded on a Bruker AC-200.1 $\left({ }^{1} \mathrm{H} 200.1 \mathrm{MHz}\right.$ and ${ }^{13} \mathrm{C} 50.3$ $\mathrm{MHz}$ ) spectrometer.

(R,S)-2-chloro ethyl 2-bromophenylacetate (1). Yield: $53 \%$. IR: 1750 and $1730\left(v_{\mathrm{C}=0}\right), 1600$ and $1475\left(v_{\mathrm{C}=\mathrm{C}}\right), 1280-1140\left(v_{\mathrm{C}-\mathrm{O}}\right) \mathrm{cm}^{-1} .{ }^{1} \mathrm{H}$ NMR $\left(\mathrm{CDCl}_{3}\right), \delta 3.65-3.71\left(\mathrm{t}, \mathrm{J}=5.7 \mathrm{~Hz}, 3 \mathrm{H},-\mathrm{CH}_{2}-\mathrm{Cl}\right), \delta 4.35-4.45$ (td, $J=2.7$ and $\left.5.7 \mathrm{~Hz}, 2 \mathrm{H},-\mathrm{OCH}_{2} \mathrm{CH}_{2} \mathrm{Cl}\right), \delta 5.40(\mathrm{~s}, 1 \mathrm{H},-\mathrm{CHBr}), \delta 7.33-$ $7.59(\mathrm{~m}, 5 \mathrm{H}, \mathrm{ArH}) .{ }^{13} \mathrm{C}$ NMR $\left(\mathrm{CDCl}_{3}\right), \delta 41.12\left(-\mathrm{CH}_{2} \mathrm{Cl}\right), 46.31(-\mathrm{CHBr})$, $65.75\left(-\mathrm{OCH}_{2}-\right), 128.79(\times 2), \quad 128.97(\times 2), \quad 129.52,135.43,168.09$ (COO). Elemental anal. calcd. for $\mathrm{C}_{10} \mathrm{H}_{10} \mathrm{O}_{2} \mathrm{BrCl}$ : C, $43.49 ; \mathrm{H}, 3.65 \%$. Found: $\mathrm{C}, 43.13 ; \mathrm{H}, 3.46 \%$.

\section{Computational Methods}

MD calculations: All MD simulations were carried out using the AMBER 9 suite of programs ${ }^{[35]}$ and the all-atom ff03 force field. ${ }^{[36,37]}$ The starting model of Burkholderia cepacia lipase was derived from the high-resolution crystal structure of $\mathrm{BCL}$ in an open conformation, available from the Protein Data Bank (PDB: 3 LIP). ${ }^{[27]}$ The calcium ion which plays a structural role in BCL was conserved in the model and appropriately parameterized according to prior work. ${ }^{[38]}$ Four $\mathrm{Na}^{+}$ 
cations were added to neutralize the protein. Models of $B C L$ mutants were constructed from wild-type BCL $X$-ray structure using the Biopolymer module of Sybyl7.3 (Tripos). MD simulations were performed in explicit solvent at water/octane interface using a procedure previously described. ${ }^{[26]}$ The simulations were carried out for a total of $20 \mathrm{~ns}$ at constant temperature and pressure conditions, using the Berendsen algorithm with a coupling constant of 2 ps for both parameters. Electrostatic interactions were calculated using the Particle-Mesh Ewald method ${ }^{[39]}$ with a non-bonded cutoff of $10 \AA$. All bonds involving hydrogen atoms were constrained with the SHAKE algorithm ${ }^{[40]}$, permitting the use of 2 -fs time steps to integrate the equations of motion. Center of mass translational and rotational motion was removed every $6000 \mathrm{MD}$ steps to avoid methodological problems described by Harvey et al. ${ }^{[41]}$ The trajectories were extended, as noted above, to $20 \mathrm{~ns}$, and conformations of the system were saved every $0.4 \mathrm{ps}$ for further analysis. Trajectories analysis was carried out using the Ptraj module of the AMBER 9 package. ${ }^{[35]}$ The root mean square deviation (RMSD) was calculated for the protein backbone atoms using least squares fit. Atomic positional fluctuations $\left(\Delta r_{i}^{2}\right)$ of backbone were calculated. A mass-weighted average value was then calculated for each residue. These parameters are related to the B-factors through the following relationship:

$B \mathrm{i}=\underline{8 \pi^{2}}\left\langle\Delta r_{\mathrm{i}}^{2}>\right.$

3

The simulated B-factors were calculated using the coordinates of the 20 ns trajectories.

Covalent docking of substrates: Starting coordinates were extracted from the crystal structure of $B$. cepacia lipase (PDB: 3LIP) to generate models of wild-type $B C L$ and its variants. Models of $B C L$ mutants were constructed by replacing in silico the target amino acid (L17 or V266) with either L17S, L17M or V266G mutation using the Biopolymer module of Insightll software package (Accelrys, san Diego). The conformation of the mutated residue side chain was optimized by manually selecting a low-energy conformation from a side-chain rotamer library. Steric clashes (van der Waals overlap) and nonbonded interaction energies (Coulombic and Lennard-Jones) were evaluated for the different side-chain conformations.

The tetrahedral intermediates of both the $R$ - and the $S$-form of the $(R, S)$-2-chloro ethyl 2-bromophenylacetate (1) were generated using Insightll. Energy minimization of each substrate was performed to generate low-energy starting conformation with suitable bond distances and angles. Starting from these initial structures, the conformational space accessible by the substrate covalently bound to the catalytic serine (Ser 87) in a tetrahedral form was investigated using the automated flexible docking program FlexX ${ }^{[42,43]}$ (Biosolveit). All parameters were set to the standard values as implemented in Version 3.1.1. FlexX uses a fast algorithm for the flexible docking of small ligands into fixed protein binding sites using an incremental construction process of the ligand ${ }^{[43]}$. The docking region was defined to encompass all protein amino acids for which at least one heavy atom was located within a $6.5 \AA$ radius sphere, whose origin was located at the centre of mass of catalytic Ser 87 . All crystallographic bound waters were removed prior to docking. Hydrogen atoms were added to the protein using standard Sybyl (Tripos) geometries. Residues were kept fixed in their crystallographic positions in all docking experiments. For each docking, the top 30 solutions corresponding to the best FlexX scores were retained.

Calculation of enantiomer trajectories: To allow the displacement of the substrates along the active site path, the covalent bond between the catalytic serine and the carbon of the substrate carbonyl function was broken to create two separate molecular entities. The hybridization of the carbonyl function was corrected from sp3 to sp2 to generate the molecular models used to search for trajectories. Starting from the docked position, enantiomer trajectories were computed using Biomove3D integrated path planner ${ }^{[16,24]}$, going from the bottom towards the entrance of the active site. During the search, both the ligand and the protein side chains were considered flexible. Atoms were modelled at $85 \%$ of their $\mathrm{vdW}$ radii.

Both the lipase and the substrate are modelled as polyarticulated mechanisms, the motion of which being restricted by geometric constraints such as steric clash avoidance between spherical atoms with van der Waals radii. The substrate is considered entirely flexible and all protein side-chains that are required to move during the computation of the pathway can do it freely. Within the ML-RRT (Manhattan-Like Rapidly-exploring Random Tree) algorithm ${ }^{[24]}$, two sets of conformational parameters -active and passive- are considered which take into account the motion of both the ligand and the protein. Molecular motions associated with each parameter type are computed in a decoupled manner. Active parameters consider the location and the internal torsions of the ligand and they are treated at each iteration of the algorithm. Passive parameters take into account the torsion angles of the protein side-chains and they only need to be treated when such side-chains hinder the motion of active or other passive parts. Therefore, the algorithm favours the ligand motion and determines the protein side-chains which must move to allow the ligand progression in the catalytic pocket of the protein. Of note, although the active and passive parts move alternately in the path computed by the ML-RRT algorithm, a randomized path smoothing post-processing is performed in the composite configuration space of all parameters, so that simultaneous motions are obtained in the final path. For each enzyme-enantiomer pair, the search tree generated by the ML-RRT algorithm was embedded in a three-dimensional data structure called voxel map. The procedure used to construct the voxel maps is identical to that described in our earlier report. ${ }^{[30]}$ Visualization and graphics were done using $\mathrm{VMD}^{[44]}$ and PyMol ${ }^{[45]}$ softwares.

Equipment: Biomove3D calculations, molecular constructions and graphic displays were performed on an Intel Pentium4 PC with a $3.2 \mathrm{GHz}$ processor. All other calculations (MD and docking) were carried out at the Computing center of Region Midi-Pyrénées (CALMIP Toulouse, France) and on the linux-cluster available at the Center for Computing Resources (CRI) of INSA-Toulouse, France.

\section{Acknowledgements}

Authors wish to thank the Computing center of Region MidiPyrénées (CALMIP, Toulouse, France) and the Center for Computing Resources (CRI) of INSA-Toulouse for providing calculation resources and support. This work was supported by the Institut des Technologies Avancées du Vivant (ITAV), Toulouse Canceropole campus.

Keywords: lipase enantioselectivity . enzymatic resolution lipases. Molecular modelling . substrate accessibility

[1] M. J. Kim, Y. Ahn, J. Park, Curr. Opin. Biotechnol. 2002, 13, 578-587.

[2] M. J. Kim, Y. K. Choi, S. Kim, D. Kim, K. Han, S. B. Ko, J. Park, Org. Lett. 2008, 10, 1295-1298.

[3] J. Nyhlen, B. Martin-Matute, A. G. Sandstrom, M. Bocola, J. E. Backvall, ChemBioChem 2008, 9, 1968-1974.

[4] O. Mc Connell, A. Bach II, C. Balibar, N. Byrne, Y. Cai, G. Carter, M. Chlenov, K. Fan, I. Goljer, H. Yanan, D. Herold, M. Kagan, M. Kerns, F. Koehn, C. Kraml, V. Marathias, B. Marquez, L. Mc Donald, L. Nogle, C. Petucci, G. Schlingmann, G. Tawa, M. Tischler, T. Williamson, A. Sutherland, W. Watts, M. Young, M.-Y.

Zhang, Y. Zhang, D. Zhou, D. Ho, Chirality 2007, 19, 658-682.

[5] F. Bordes, E. Cambon, V. Dossat-Letisse, I. André, C. Croux, J. M. Nicaud, A. Marty, ChemBioChem 2009.

[6] R. Kato, H. Nakano, H. Konishi, K. Kato, Y. Koga, T. Yamane, T. Kobayashi, H. Honda, J. Mol. Biol. 2005, 351, 683-692.

[7] Y. Koga, K. Kato, H. Nakano, T. Yamane, J. Mol. Biol. 2003, 331, 585-592.

[8] T. Schulz, J. Pleiss, R. D. Schmid, Protein Sci., 2000, 9, 1053-1062.

[9] X.-G. Li, M. Lähitie, M. Päiviö, L. T. Kanerva, Tetrahedron: Asymmetry 2007, $18,1567-1573$

[10] K. Dąbkowska, K. W. Szewczyk, Biochem. Eng. J. 2009, In Press. 
[11] D. Guieysse, J. Cortes, S. Puech-Guenot, S. Barbe, V. Lafaquiere, P. Monsan, T. Simeon, I. André, M. Remaud-Simeon, ChemBioChem 2008, 9 , 1308-1317.

[12] D. Guieysse, C. Salagnad, P. Monsan, M. Remaud-Siméon, V. Tran, Tetrahedron: Asymmetry 2003, 1807-1817.

[13] M. S. Apaydin, D. L. Brutlag, C. Guestrin, D. Hsu, J. C. Latombe, C. Varma, J. Comput. Biol. 2003, 10, 257-281.

[14] A. P. Singh, J. C. Latombe, D. L. Brutlag, Proc. Int. Conf. Intell. Syst. Mol. Biol. 1999, 252-261.

[15] J. Cortes, T. Simeon, M. Remaud-Siméon, V. Tran, J. Comput. Chem. 2004, 25, 956-967.

[16] J. Cortes, T. Simeon, V. Ruiz de Angulo, D. Guieysse, M. Remaud-Siméon, V. Tran, Bioinformatics 2005, 21 Suppl 1, i116-125.

[17] S. Kirillova, J. Cortes, A. Stefaniu, T. Siméon, Proteins: Struct., Funct. and Bioinf. 2008, 70, 131-143.

[18] X. Tang, B. Kirkpatrick, S. Thomas, G. Song, N. M. Amato, J. Comput. Biol. 2005, 12, 862-881.

[19] A. Enosh, B. Raveh, O. Furman-Schueler, D. Halperin, N. Ben-Tal, Biophys J. 2008, 95, 3850-3860.

[20] B. Raveh, A. Enosh, O. Schueler-Furman, D. Halperin, PLoS Comput. Biol. 2009, 5, e1000295.

[21] N. M. Amato, K. A. Dill, G. Song, J. Comput. Biol. 2003, 10, 239-255.

[22] G. Song, S. Thomas, K. A. Dill, J. M. Scholtz, N. M. Amato, Pac. Symp.

Biocomput. 2003, 240-251.

[23] V. Ruiz de Angul, J. Cortés, T. Siméon, Proc. Robotics: Science and Systems 2005.

[24] J. Cortés, L. Jaillet, T. Siméon, IEEE Int. Conf. Robotics and Automation

2007

[25] S. Puech-Guenot, V. Lafaquière, D. Guieysse, L. Landric-Burtin, P. Monsan,

M. Remaud-Siméon, J. Biomol. Screen. 2008, 13, 72-79.

[26] S. Barbe, V. Lafaquière, D. Guieysse, P. Monsan, M. Remaud-Siméon, I.

André, Proteins: Struct., Funct. and Bioinf. 2009.

[27] J. D. Schrag, Y. Li, M. Cygler, D. Lang, T. Burgdorf, H. J. Hecht, R. Schmid,

D. Schomburg, T. J. Rydel, J. D. Oliver, L. C. Strickland, C. M. Dunaway, S. B.

Larson, J. Day, A. McPherson, Structure 1997, 5, 187-202.

[28] K. K. Kim, H. K. Song, D. H. Shin, K. Y. Hwang, S. W. Suh, Structure 1997 $5,173-185$

[29] S. M. La valle, J. J. Kuffner, Algorithmic and computational robotics: New

Directions (WAFR2000) 2001, 293-308.

[30] J. Cortés, S. Barbe, M. Erard, T. Siméon, IEEE Int. Conf. Robotics and

Automation 2009, In press.

[31] J. Ottosson, L. Fransson, K. Hult, Protein Sci. 2002, 11, 1462-1471.

[32] J. Ottosson, J. C. Rotticci-Mulder, D. Rotticci, K. Hult, Protein Sci 2001, 10,

1769-1774.

[33] E. Y. Lau, K. Kahn, P. A. Bash, T. C. Bruice, Proc. Natl. Acad. Sci. USA

2000, 97, 9937-9942.

[34] D. Guieysse, C. Salagnad, P. Monsan, M. Remaud-Simeon, Tetrahedron: Asymmetry 2003, 14, 317-323.

[35] D. A. Case, T. E. Darden, I. T. E. Cheatham, C. L. Simmerling, J. Wang, R. E. Duke, R. Luo, K. M. Merz, D. A. Pearlman, M. Crowley, R. C. Walker, W

Zhang, B. Wang, S. Hayik, A. Roitberg, G. Seabra, K. F. Wong, F. Paesani, X.

Wu, S. Brozell, V. Tsui, H. Gohlke, L. Yang, C. Tan, J. Mongan, V. Hornak, G.

Cui, P. Beroza, D. H. Mathews, C. Schafmeister, W. S. Ross, P. A. Kollman,

AMBER 9, University of California, San Francisco 2006.

[36] Y. Duan, C. Wu, S. Chowdhury, M. C. Lee, G. Xiong, W. Zhang, R. Yang, P.

Cieplak, R. Luo, T. Lee, J. Caldwell, J. Wang, P. Kollman, J. Comput. Chem.

2003, 24, 1999-2012.

[37] M. C. Lee, Y. Duan, Proteins 2004, 55, 620-634

[38] J. Aaqvist, J. Phys. Chem. 1990, 94, 8021-8024.

[39] U. Essmann, L. Perera, M. L. Berkowitz, T. Darden, H. Lee, L. G. Pedersen, J. Chem. Phys. 1995, 103, 8577-8593.

[40] J. P. Ryckaert, G. Ciccotti, H. J. C. Berendsen, J. Comput. Phys. 1977, 23, 327-336.

[41] S. C. Harvey, R. K. Z. Tan, I. T. E. Cheatham, J. Comput. Chem. 1998, 19,

726-740.

[42] B. Kramer, M. Rarey, T. Lengauer, Proteins: Struct., Funct. and Genetics 1999, 37, 228-241.

[43] M. Rarey, B. Kramer, T. Lengauer, G. Klebe, J. Mol. Biol. 1996, 261/3, 470489.

[44] W. Humphrey, A. Dalke, K. Schulten, J. Molec. Graphics 1996, 14, 33-38.

[45] W. L. DeLano, The PyMOL Molecular Graphics System on World Wide Web http://www.pymol.org. 2002. 